\title{
La revolución cognitiva en la sociedad actual: nuevos retos educativos.
}

\author{
María José Hernández Serrano \\ mjhs@usal.es \\ Margarita González Sánchez \\ mgsalusal.es \\ Universidad de Salamanca
}

\section{Los cambios de la sociedad actual.}

En la actualidad nos encontramos inmersos en un nuevo orden social generado a partir de diferentes cambios. Estamos ante una verdadera revolución tecnológica e informacional (Castells, 1997), que lejos de considerarse como una mera transición u optimización de métodos y actividades, está derivando en la aceptación de un nuevo imperativo social, donde las modificaciones son lo suficientemente significativas como para marcar una discontinuidad histórica frente a etapas pasadas.

Este momento presente se caracteriza por la rapidez con la que se han extendido las innovaciones, por su consistencia y por las repercusiones decisivas de los cambios, que contribuyen a innovar sobre las prácticas sociales más tradicionales.

Todo esto está generando nuevos retos para todos los sistemas sociales en los que participa el individuo, con modificaciones relevantes en cuanto a los aspectos socioculturales, socioeconómicos, políticos, educacionales, etc.

Sin embargo, es preciso considerar que además de la afectación en los diferentes ámbitos o macrocontextos sociales, estos cambios están trastocando también las microestructuras interpersonales de los individuos. En primera instancia, porque estos cambios contribuyen a la transformación social y con ello, a la transformación de las representaciones que los sujetos tienen de la sociedad. Y en segundo lugar, más concretamente, porque los principales cambios se relacionan con modificaciones en materia de información, objeto indispensable de la actividad intelectual de los sujetos.

La información adquiere nuevas características debido a las posibilidades de las nuevas tecnologías en línea en términos de sobreabundancia, hipermedialidad, accesibilidad y diversidad. Y son principalmente estas cuatro propiedades, además de otras muchas, las que están exigiendo otros modos de relación con la información, demandando de los sujetos nuevas formas para acceder, procesar y generar conocimiento, en definitiva, nuevas competencias cognitivas, que hacen preciso ajustar las habilidades tradicionales de los sujetos para operar con la información.

A pesar de todas estas modificaciones, no existe una clara visibilidad que impulse el desarrollo de nuevas destrezas y aptitudes para el manejo de la información. La visibilidad de estos cambios a nivel cognitivo se encuentra aún en un nivel latente.

\section{Visibilidad de los cambios cognitivos.}

No hay duda de que en la nueva sociedad se recrean nuevos procesos de socialización, de enculturación o de educación. Somos conscientes de la revolución "de las comunicaciones", "de las tecnologías", "de la información”, y sus repercusiones en numerosos ámbitos sociales, desde el financiero hasta el comercial, el sanitario o incluso el educativo. Sin embargo, apenas se habla de las repercusiones a nivel micro, de las modificaciones cognitivas que se están generando en los sujetos. 
No son muchos los autores que se aventuran firmemente a establecer las bases de la revolución cognitiva actual. El propio Castells, considerado uno de los principales eruditos de la sociedad actual, apenas deja entrever las implicaciones que esta nueva sociedad y las tecnologías preponderantes del momento suponen para las estructuras y los procesos mentales de los individuos. De forma general, afirma que asistimos a un momento de revolución, momento inductor de discontinuidad en la base material de la economía, la sociedad y la cultura, sin profundizar en otras transformaciones concretas de lo personal. Y a pesar de que señala que esta revolución "se dirige a la modificación de los procesos, más que a la inducción de nuevos productos” (1997: 57), tan sólo afronta el tema del procesamiento de la información para referirse a la cualidad de la mente humana como "fuerza productiva directa" del sistema económico, el cual opera y subsiste a partir de la generación y el intercambio de información y conocimiento. El propio autor se cuestiona como el empleo se está concentrando en el procesamiento de la información desde el punto de vista de la economía, las empresas y las tecnologías encargadas de este cometido, sin indagar apenas en las repercusiones que este mismo procesamiento de la información comporta a nivel personal en cuanto a la adquisición de nuevas formas y estrategias operativas de procesamiento.

Y es que, como concluye Navarro, la mayor parte de la gente informada estaría dispuesta a reconocer que "nuestra sociedad está experimentando cambios a nivel comunicativo o informativo, incluso económico, no percibiéndose con igual nitidez una revolución en la esfera de lo propiamente cognitivo" (2002: 120).

Pero lo cierto es que se están produciendo modificaciones cognitivas, respecto a la forma de acceder y procesar la información, debido a la aparición de nuevas tecnologías informativas. Y éste es un matiz que no resulta precisamente novedoso, ya que en otros momentos históricos anteriores, también bajo la influencia de ciertas tecnologías imperantes, se ha estudiado cómo la adquisición de diversos sistemas simbólicos y el uso de determinadas herramientas culturales tienen efectos decisivos en los modos de pensamiento y funcionamiento psicológico (Del Río, 1993).

Es fácil advertir cómo cada vez que surge una nueva tecnología comunicacional (como la palabra, el libro impreso o las TICs) no sólo se aumenta la accesibilidad de los sujetos a la información, sino que además se demandan nuevas capacidades en los sujetos relacionadas con la apropiación de los nuevos códigos que la tecnología proyecta. Estas consideraciones han sido objeto de análisis de numerosos autores, desde Ong a Olson, hasta McLuhan y De Kerckhove, que han venido investigado el impacto de las diferentes tecnologías tanto en lo que se refiere a la estructura, como al contenido de nuestras mentes.

Por su parte McLuhan analizó cómo se ha promovido un desarrollo sensitivo y cerebral diferente en base a las tecnologías que han operado en cada sociedad. En la etapa oral o pre-alfabética se desarrollaron las funciones asociadas al hemisferio derecho (sintetizador), más cualitativas y holísticas, y en la etapa alfabética o lecto-escritora las del hemisferio izquierdo (analizador), relacionadas con las funciones para el cálculo, el habla, la escritura y las capacidades lingüísticas generales (McLuhan, 1960: 63).

En la sociedad actual, con las Tecnologías de la Información y la Comunicación -que según McLuhan (1990), son del hemisferio derecho en sus normas y en su operación-, se van a precisar nuevas formas de procesamiento que van a requerir, según el autor, del trabajo conjunto de los dos hemisferios, sin exclusividad o tendencias hacia ninguno, sino más bien, y de forma especial hacia el desarrollo de las funciones asociadas al lóbulo frontal, donde se llevan a cabo las funciones superiores de planificación, control y ejecución, habilidades que resultarán esenciales en la actualidad.

Los cambios tecnológicos, por tanto, son decisivos para el funcionamiento cognitivo de los sujetos. Y, pese a que en la actualidad apenas se haya abordado suficientemente esta cuestión, ni se hayan asumido de forma explícita estos cambios, a nuestro modo de ver, empiezan a ser frecuentes ciertas aserciones que redundan en advertir cómo en la actualidad se está sucediendo una renovación cognitiva. Una renovación que va a comportar una serie de posibilidades para los sujetos, pero que 
también va a demandar de los mismos ciertas habilidades y destrezas.

Expondremos a continuación las principales aportaciones que suponen las tecnologías informacionales a nivel cognitivo, para delimitar seguidamente los retos generados en torno a las mismas, que deberán transformarse coherentemente en nuevas competencias a adquirir por los sujetos.

\section{Aportaciones básicas para el sistema cognitivo.}

Como venimos exponiendo, la llegada de una nueva tecnología genera ciertas alteraciones en las disposiciones cognitivas de los sujetos, en cuanto a nuevos modos de procesamiento de la realidad y de la información misma.

Si nos centramos en cómo se desarrolla la llegada de nuevas tecnologías, es preciso tener en cuenta que en su origen, las tecnologías son inventadas para resolver ciertas limitaciones que puedan promover el avance de las sociedades y la próspera evolución de los sujetos. La nueva tecnología pretende introducir mejoras tanto en los modos de producción como de comunicación, afectando a los diferentes ámbitos sociales. Por ello, y en lo que se refiere al dominio cognitivo, las tecnologías también van a permitir optimizar y ampliar ciertas capacidades intelectuales (Marqués, 2000). Esta ampliación de las capacidades está vinculada con el archicitado pensamiento McLuhaniano de que las tecnologías o los artefactos son extensiones para el individuo. Desde este pensamiento, se entiende que las tecnologías multiplican las posibilidades de los sujetos para la realización de diversos cometidos y facilitan nuevas formas de actuación e interactuación con algún tipo de reducción en cuanto a costes, espacios o tiempos, respecto a etapas pasadas.

En consonancia con esto, en la actualidad se empieza a deliberar en torno a nuevas formas de prolongación relacionadas con las tecnologías presentes. Así, algunos autores han encontrado algunas extensiones cognitivas, que incrementan la actividad mental de los sujetos en diferentes procesos. Estebanell y Ferrés (2001) exponen cómo las posibilidades tecnológicas permiten amplificar y exteriorizar algunas funciones cognitivas como la memoria (bases de datos, hiperdocumentos), la imaginación (simulaciones), o la percepción (captadores numéricos, telepresencias, realidades virtuales...). De todas éstas, la contribución más relevante, señalada por numerosos autores (De las Heras, 1991; Tiffin y Rajasingham, 1997 y Mattelart, 2002), se refiere a la idea de "memoria auxiliar" en cuanto a la prolongación de nuestra memoria biológica. Nuestra memoria biológica queda limitada frente a las posibilidades que ofrecen las tecnologías. Así, mientras nosotros seguimos teniendo los mismos recursos y las mismas capacidades cognitivas limitadas para el procesamiento de la información, proliferan las herramientas para almacenar, transmitir y acceder a la información (Sancho 2001), que conformarán nuestra memoria auxiliar. Y esto invita a reflexionar sobre la posibilidad de utilizar estas herramientas tecnológicas actuales para superar nuestras propias limitaciones.

En la actualidad, Internet se está convirtiendo en nuestra memoria auxiliar, debido principalmente a su ilimitada capacidad. A la Red acudimos cuando necesitamos información sobre algo, porque es posible acceder a una vasta cantidad de información variada en cualquier momento. Y esto hace pensar en Internet como una memoria exenta (De las Heras, 1991) o colectiva (Mattelart, 2002), que amplía las posibilidades de almacenamiento y recuperación de información que poseen biológicamente los individuos.

Sin embargo, esta memoria auxiliar también cuenta con una serie de inconvenientes para los sujetos, que dificultan su óptimo aprovechamiento y que exigen además nuevas estrategias cognitivas y metacognitivas para operar con la información.

Uno de los principales inconvenientes se refiere a la dificultad para gestionar el amplio volumen informativo, ya que Internet es un medio de fácil publicación y distribución que no ofrece resistencia ante informaciones de escasa calidad, ni está sujeta a clasificaciones que permitan ordenar los contenidos distribuidos. 
La Red desarrolla una función editora sin apenas intermediación, capacitando a los sujetos para publicar directamente su propia información. Y al no existir filtros que delimiten qué tipo de información es susceptible de publicación, en cuanto al contenido, a los fines o a los destinatarios, todo se publica porque, en principio, todo puede ser valioso. Y no sólo esto, sino que además toda esta información disponible no puede clasificarse, porque no se sabe ni cuánta ni en torno a qué temas se genera información cada día. Esto está evidenciando un desorden informativo que no facilita la recuperación precisa de información.

De nada sirve que en esta gran memoria exista mucha información diversa y accesible, si no se garantiza cierta calidad, y sobretodo, si no se sabe cómo acceder a lo que realmente se necesita. Y esto es algo que afecta directamente a los sujetos, puesto que saben que pueden encontrar información, pero no saben cómo buscarla, navegando horas y horas hasta llegar a la información que desean, o lo que es peor, topándose en algunas ocasiones con contenidos no deseados, ilícitos o ilegales, y sorteando publicidad abrumadora.

Esto hace difícil el camino de acceso real a la información, teniendo que adivinar el sujeto qué caminos pueden llevarle acertadamente hasta su objetivo. Alcanzar de forma efectiva la información deseada es un proceso costoso que requiere tiempo y esfuerzo, para poner en marcha ciertas estrategias que permitan localizar acertadamente la información.

Otro de los inconvenientes de esta memoria auxiliar se refiere al dinamismo y a la constante actualización de Internet. Dados los reducidos costes de edición y publicación, es posible modificar y reeditar la información de forma rápida, para hacerla de nuevo disponible. Esta factible actualización -ya que de hecho no toda la información está actualizada, ni se actualiza- dota a la información de un carácter abierto, vivo, no estático, capaz de reconfigurarse para adaptarse a las particularidades del momento. Esto también conlleva a que mucha de la información desaparezca y sea relevada por nueva, lo que indica que la información tiene un ciclo de vida. Todo esto se traduce de nuevo en la dificultad para localizar una información dentro de esta gran memoria auxiliar, que al no permanecer estable, no garantiza que al acceder por segunda vez a una misma información se encuentre en el mismo lugar o no haya sufrido modificaciones.

Precisamente esta constante evolución y modificación imposibilita el registro de toda la información existente. No es posible conocer cuánta información existe sobre un tema para evitar contradicciones o redundancias, haciendo frecuente la duplicidad de información. Esto nos lleva a otro de los inconvenientes de esta gran memoria que hace referencia a criterios de calidad, veracidad o autenticidad.

La libertad que permite Internet y la ausencia de filtros de calidad están poniendo en cuestión su fiabilidad informativa. De este modo, una considerable porción de información es de naturaleza dudosa, de escasa exactitud, contradictoria, nociva o ciertamente mediocre. Poca de la información contenida en Internet ha sido organizada, catalogada o evaluada bajo criterios científicos y de relevancia científica. Y a veces, son las páginas menos accesibles o restringidas las que suelen poseer mayores índices de calidad.

Todo esto dificulta sin duda la utilización de Internet como memoria externa, ya que no sólo se complica el proceso de localización de la información que se necesita, sino que además es preciso analizar con cautela el contenido de lo que se recupera, para valorar tanto el origen, la presentación y la veracidad, como la capacidad de contraste con información similar. Estas necesidades van a derivar en una serie de retos para los individuos, respecto a nuevas estrategias y habilidades para operar con la información de esta gran memoria auxiliar.

\section{Principales retos cognitivos para los individuos.}

A pesar de los inconvenientes que presenta Internet en cuanto memoria auxiliar, las posibilidades informacionales que ofrece son suficientemente relevantes como para reflexionar en torno a un 
conjunto de habilidades esenciales, que capaciten a los sujetos y puedan equilibrar todas estas limitaciones.

Es preciso matizar previamente, que algunas de estas limitaciones son connaturales al propio medio, han permanecido desde sus orígenes hasta la actualidad, por lo que seguirán siendo características. Pero otras son susceptibles de modificación, y de hecho se están sucediendo numerosas investigaciones y proyectos que tratan de estudiar algunos de estos problemas, haciendo cada vez más intuitivas las tecnologías y optimizando sus posibilidades. Este es el caso de las innovaciones en materia de semántica, como la Web Semántica, que posibilitará la descripción, definición, relación y contextualización de la información (Hernández y otros, 2005). También otras técnicas y especializaciones científicas para el tratamiento de los problemas de desorganización y desestructuración de la información contenida en la Web, como la Arquitectura de la Información (Baeza y otros, 2004) - para cuestiones de diseño y usabilidad- y la Minería de Datos -para descubrir relaciones entre datos y establecer información a partir de otra información (García, 2003).

A pesar de que estos avances dan cuenta de que las tecnologías se optimizan a sí mismas para resolver problemas relacionados con la ordenación y manejabilidad de la información y de sus enlaces, no resuelven la totalidad de los problemas. En parte, como ya señalábamos anteriormente, porque Internet posee una serie de características connaturales difíciles de corregir o eliminar. Inconvenientes como la falta de control sobre los contenidos distribuidos, la sobreabundancia, o el problema de la calidad de la información seguirán manteniéndose. Esto significa que para contrarrestar estas limitaciones los sujetos deberán aplicar diversas estrategias cognitivas, que supondrán ciertos retos respecto a la forma usual de tratar con la información.

Las estrategias necesarias se van a centrar principalmente en el uso de la memoria auxiliar que ofrece Internet. Así, del mismo modo que utilizamos estrategias para acceder, procesar y recuperar información en nuestra memoria biológica, también accederemos a Internet poniendo en marcha diversas estrategias de búsqueda, de selección, de conexión, de interpretación, etc.

Estas estrategias de usabilidad de la memoria auxiliar van a ser muy diferentes de las que tradicionalmente han venido desarrollando los sujetos, por lo que las transformaciones necesarias van a implicar nuevos desafíos tanto para los individuos, como para las instituciones y agentes encargados de instruirlos, como veremos más adelante.

En este sentido, ya no va a ser tan necesario que los individuos adquirieran un cuerpo de conocimientos finitos, tanto como que desarrollasen estrategias para aprender a aprender, y para saber buscar, valorar y gestionar el amplio volumen de información. Por una parte, la sobreinformación desborda las capacidades de asimilación del ser humano, exigiéndole una valoración constante de información (Picardo, 2002). El volumen de información disponible en la actualidad, desborda las capacidades de asimilación y procesamiento de los individuos (Area, 2001), por lo que seguir pretendiendo desarrollar las habilidades cognitivas tradicionales de acumulación y de reproducción de la información, resulta hoy en día algo inverosímil. Se hace necesario formar a los individuos en otro tipo de habilidades para la recuperación, el análisis y la comunicación de información, relacionadas con procesos cognitivos superiores, a través de los cuales puedan valorar y contrastar la información de esta memoria auxiliar.

Y por otra parte, si deja de resultar relevante y viable la acumulación continuada de información, ya que gracias a la memoria auxiliar es posible acceder a prácticamente todo el contenido disponible, empezarán a tomar importancia otras habilidades más complejas como el hecho de saber buscar información, discriminar entre diversas fuentes, localizar y recuperar información relevante, organizarla, evaluarla, etc., tareas esenciales para procesar información y generar conocimiento.

También, como hemos señalado, se pone en cuestión la fiabilidad y validez de la información, lo que va a requerir de los individuos nuevas actitudes y habilidades para valorar y crear información. La sobreabundancia y diversidad informativa a la que están expuestos los individuos exigirá de los 
mismos ciertas estrategias para priorizar la información relevante, y para valorar su origen o su calidad.

Por otra parte, la multiplicidad de medios (texto, sonido, imagen, vídeo, animación, gráfico, mapa conceptual...) que es posible combinar para la generación y presentación de información en Internet requerirá nuevos conocimientos y estrategias para la descodificación de los diferentes lenguajes, así como el aprendizaje de nuevos códigos, sobre todo icónicos y visuales que permitan la creación de producciones propias a través de estos mismos medios (Correa, 2001).

Todas estas estrategias y habilidades necesarias en esta revolución cognitiva actual, van a presentar como venimos advirtiendo nuevos retos o posibilidades para el funcionamiento cognitivo. Estos retos cognitivos generarán nuevas exigencias que deberán responderse desde el ámbito educativo para posibilitar el afrontamiento de dichos cambios con la prontitud y la relevancia que merecen. El sistema educativo deberá desarrollar nuevas competenciasy nuevas formas de alfabetización de acuerdo a las nuevas exigencias que la misma sociedad demanda de los individuos.

Veamos a continuación cómo es percibida esta revolución cognitiva desde el ámbito educativo y cómo es posible afrontar los cambios que se sustraen de la misma.

\section{Retos educativos: las competencias cognitivas.}

Desde esta "revolución de lo cognitivo", ciertamente silenciosa (Aguilera, 2000), por las pocas contribuciones o hallazgos manifiestos, empiezan a demandarse, como venimos exponiendo, algunas modificaciones en el funcionamiento cognitivo de los sujetos relacionadas con nuevas formas de pensar, de elaborar y de asimilar la información. A pesar de que se ha empezado a reflexionar en torno a la visibilidad de estos cambios, todavía no se puede asegurar que se perciban con nitidez, lo que dificulta tanto la claridad de los retos, como el afrontamiento de responsabilidades por parte de los diferentes ámbitos.

Debido a esto, muchas de las estrategias y habilidades cognitivas que son necesarias en la actualidad se están desarrollando de modo implícito, como un efecto indirecto derivado de la utilización de las nuevas tecnologías, cercano a lo que Monereo (2005) expone como "la mente virtual de los nativos tecnológicos". Las nuevas generaciones de individuos que crecen y se desarrollan en torno a las nuevas tecnologías se encuentran más familiarizados, mediatizados y sobretodo preparados para desenvolverse y progresar en y con los nuevos medios telemáticos. Estos nativos tecnológicos adquieren mayor facilidad para procesar información discontinua (Sanz, 2005) e interconectada, aprenden a resolver sus necesidades informativas a través del acceso y la búsqueda de información en Internet y aprenden a relativizar la importancia de lo que leen (Monereo, 2005). En definitiva, los sujetos se vuelven más estratégicos modificando sus formas de procesamiento cognitivo, y en ocasiones siendo a penas conscientes de que cada vez son más competentes para la resolución eficiente de las tareas y para el desempeño tecnológico.

Todo esto indica que aún no se ha abordado de forma explícita la asunción de estos cambios cognitivos. Quizás, y en parte, por que se piense que los procesos cognitivos son demasiado subjetivos y con frecuencia difíciles de observar y de medir, como para estandarizarlos a nivel de competencias generales para todos los individuos. Y esto tal vez sea lo que conlleva a que todas estas habilidades se generen en un contexto desprovisto de orientaciones o planificaciones precisas.

Sin embargo, para el desarrollo óptimo de nuevas formas de procesamiento cognitivo, y para prevenir o paliar ciertos peligros y riesgos relacionados con la inseguridad del medio, se hace necesario que los individuos adquieran de forma explícita una serie de competencias y una alfabetización tecnológica e informacional (Hernández, 2005), un conjunto de habilidades y actitudes que les permitan no sólo aprovechar las potencialidades informativas del medio, sino acceder y procesar la información de forma eficiente. 
Por ello pensamos, que al ámbito educativo le corresponde un importante papel en la asunción de estos cambios, dado su compromiso sobre la formación de los individuos. En concreto a la escuela, que se ha configurado desde sus inicios como el instrumento principal -al menos en los países desarrollados- de estandarización de un conjunto de conocimientos y habilidades básicas para la emancipación y el desarrollo colectivo de los individuos (Area, 2002). Esa, esta escuela, que se ha organizado desde hace siglos como un sistema de transmisión de conocimientos útiles para vivir en la sociedad. El conjunto de habilidades y conocimientos básicos se aprendían en la etapa de escolarización obligatoria y se consideraban de alguna manera finitos, porque culminaban con dicha etapa (Majo, 2000). Es decir, los conocimientos eran limitados y podían trasmitirse de unos individuos a otros. Y estos mismos principios de limitación y transmisión son los que han fundamentado durante mucho tiempo la labor reproductora de la escuela y las funciones de sus agentes, desde los métodos expositivos de los profesores, hasta la tarea receptora del alumno.

Además, este modelo se ha visto favorecido porque la gran mayoría del conocimiento se almacenaba en textos, conformando una cultura escrita, donde el discurso oral de los educadores era el principal canal de transmisión y además, las dos habilidades básicas por excelencia, en las que se instruía a los sujetos, eran la lectura (para la comprensión) y la escritura (para la expresión a través de textos).

Pero hoy en día hemos pasado de "un mundo en papel" - como gusta llamarlo a Olson (1998) - a un mundo gobernado por múltiples lenguajes además del textual, el audiovisual y el informático. Y esto unido a la acumulación y el desbordamiento de los contenidos disponibles ha contribuido a la necesidad de preparar a los individuos para la comprensión de la información en los diferentes lenguajes y soportes, nuevas habilidades que confrontan con las que tradicionalmente se han venido desarrollando en la escuela.

Es evidente, que durante mucho tiempo la escuela ha promovido un conjunto de habilidades para la interpretación y la expresión de un lenguaje lineal, lo que ha generado un modo de procesamiento secuencial y un aprendizaje receptivo y asociativo. También un tipo de pensamiento pasivo, como expone DeKerckhove aprendiendo a pensar "escuchando un tipo de habla formal, entrenado y estructurado del profesor" (1995:136). Sin embargo, en la actualidad los lenguajes audiovisuales han superado a la oralidad y textualidad tradicionales, debido a que las nuevas tecnologías existentes aglutinan los diferentes modos de lenguaje (Correa, 2001). Por esta razón pensamos necesario que la escuela aproveche las oportunidades cognitivas que estos medios aportan, en cuanto a las posibilidades para representar el conocimiento a partir de diferentes lenguajes, formando individuos capaces de decodificar, comprender y crear expresiones y representaciones que integren todos estos lenguajes.

Además, la evolución continua de los entornos tecnológicos, económicos y sociales precisa una actualización permanente del saber (Arbués y Tarín, 2000). Esto hace preciso asumir, más que nunca, que los individuos deben educarse a lo largo de la vida, ya que los conocimientos se modifican constantemente y tienen un ciclo de vida concreto, no siendo hoy suficiente con la formación recibida en un único ámbito, o en un momento concreto de la vida. Aprender a lo largo de la vida implica adaptarse a vivir en un mundo en constante cambio, donde lo importante es adquirir estrategias para aprender a aprender, y adquirir los conocimientos en el momento en el que se necesiten (Majó, 2000).

El hecho de que en la actualidad se disponga, de forma inmediata y permanente, de grandes cantidades de información a través de Internet, contribuye, por un lado, a que sean más importantes los procesos de descubrimiento de información relevante, que la apropiación de la información misma como objetivo final (De Kerckhove, 1995; Majó, 2000); y por otro, a que se reduzca el protagonismo de los profesionales de la educación, en especial de los profesores, como únicos poseedores y transmisores de conocimientos, dadas las múltiples vías de acceso y de transmisión de información existentes en la actualidad. Hoy en día lo que los conocimientos que se aprehenden en la escuela pueden ser contrastados, ampliados y/o modificados a través de información procedente de Internet. Y aunque, como hemos comentado, debemos mostrar cierto recelo hacia la información que proviene de ella, ya que puede no ser fiable, actualizada o acertada, no podemos negar sus aptitudes como medio 
informativo de pluralidad temática, en la que encontrar información para cualquier necesidad o propósito.

En este sentido hay que precisar que acceder y tener información no se traduce directamente en la generación de conocimientos o en el aprendizaje. De esta manera, posee conocimiento no aquel que tiene información, sino aquel que sabe qué hacer con la información (Briones, 2002),

independientemente de si su recuperación se ha realizado en Internet, o en otra fuente de información.

En la línea de lo que venimos exponiendo, lo importante no va a ser transmitir una información, puesto que existen muchos medios disponibles desde los que se puede acceder a mucha información, lo realmente importante será saber cómo encontrar información para procesarla, utilizarla, y relacionarla con la información que ya se posee. Porque información es lo que tenemos, seguramente en mayor cantidad, diversidad o calidad en cuanto a posibilidades de acceso respecto a otras sociedades pasadas, pero el conocimiento es lo que queremos llegar a conseguir. Y puesto que para que exista conocimiento es necesario ayudar a los individuos a procesar y valorar la información existente, consideraremos que la escuela debe desarrollar una tarea esencial mediando en el desarrollo socio-cognitivo de los alumnos.

En consecuencia, entendemos que la escuela debe considerar los cambios cognitivos generados en la nueva sociedad, para que los individuos desarrollennuevas estrategias y habilidades cognitivas, del mismo modo que, en otros momentos sociales y respecto de otras tecnologías, esta institución adquirió la responsabilidad de asumir la formación competencial de los individuos.

Todo esto, generará sin duda múltiples retos para el sistema educativo, cambiando los roles tanto del profesor como del alumno. Las competencias básicas se centrarán en la enseñanza y el aprendizaje para la resolución de demandas informativas, en el acceso a la información digital utilizando los recursos pertinentes, en el análisis de la información recuperada, etc., y todos estos serán nuevos procesos que confrontarán con las representaciones tradicionales de lo que durante mucho tiempo se ha entendido como labor del profesor o del educador, identificada con métodos expositivos; y del mismo modo la tarea del alumno, quién ha tenido un rol esencialmente receptivo y pasivo, y cuyo principal acceso a la información y al conocimiento se realizaba a través del profesor, como mediador del aprendizaje.

Además de la transformación de los métodos y los roles de los agentes, también se modificarán los métodos y los marcos teóricos que fundamentan las destrezas requeridas a los sujetos, enfatizándose más la adquisición de procesos que el logro de los resultados. Puesto que se puede recurrir de forma constante y permanente a la información, la importancia radicará en el proceso de acceso que permita la búsqueda y localización de la información para recurrir a ella en momentos posteriores.

En definitiva, los cambios cognitivos brindarán la oportunidad de reformular y repensar los modos tradicionales del sistema educativo (Pérez, 2004), para ajustar esta institución a las demandas de la revolución actual. Mientras hemos venido desgastando nuestra memoria biológica con meras tareas de memorización y de asociación, en la actualidad, gracias a las posibilidades informacionales de Internet, se nos presenta la posibilidad de desarrollar nuevas funciones cognitivas, y de potenciar las funciones cognitivas superiores. Es de este tipo de implicaciones de las que deberá hacerse eco la educación, más concretamente, debe responsabilizarse para que la revolución cognitiva que hemos presentado se desarrolle adecuadamente y en sintonía con los cambios presentes.

\section{Bibliografía}

AGUILERA, R. (s.f.): El procesamiento humano de la información. Consultado el 2 de Febrero de 2005 en http://academico.uno.mx/amancinas/proceso_ea/Lecturas/Cognoscitivismo.pdf

ARBUÉS, M. T. y TARÍN, LL. (2000): Aprender a lo largo de la vida y las nuevas tecnología, en DUART, J. M. y SANGRÁ, A. (Eds.): Aprender en la virtualidad. Barcelona, Gedisa, 51-60. 
AREA, M. (coord.) (2001): Educar en la sociedad de la información. Bilbao, Desclée de Brouwer.

AREA, M. (2002): Igualdad de oportunidades y nuevas tecnologías. Educar, 29, 55-65.

BAEZA, R., RIVIERA, C. y VELASCO, J. (2004): Arquitectura de la información y usabilidad en la Web. El profesional de la información, 13 (3), 168-178.

BRIONES, L. (2002): Demandas de la Sociedad del Conocimiento. Pensamiento Educativo, política y gestión de la educación, 31, 390-427.

CASTELLS, M. (1997): La era de la información: economía, sociedad y cultura. Vol.1: La sociedad red. Madrid, Alianza.

CORREA, R. I. (2001): La sociedad mesmerizada: medios, nuevas tecnologías y conciencia crítica en educación. Huelva, Universidad de Huelva.

DE KERCKHOVE, D. (1995): La piel de la cultura. Investigando una nueva realidad electrónica. Barcelona, Gedisa.

DE LAS HERAS, A. R. (1991): Navegar por la información. Madrid, Fundesco.

DEL RÍO, P. (1993): La respuesta a la cultura de los múltiples lenguajes. Cuadernos de Pedagogía, 216, 32-34.

ESTEBANELL, M. y FERRÉS, F. (2001): Espacios virtuales y educación a distancia. En AREA, M. (Ed.) Educar en la sociedad de la información. Bilbao, Desclée de Brouwer, 325-358.

GARCÍA, L. (2003): Extraer y visualizar información en Internet: el web mining. Gijón, Trea.

HERNÁNDEZ, M. J. (2005): El acceso a la información digital: Mediación en la generación de conocimientos y nuevas competencias para la educación del siglo XXI. Trabajo de Grado no publicado, Departamento de Teoría e Historia de la Educación, Universidad de Salamanca.

HERNÁNDEZ, M. J., LÓPEZ, C., GONZÁLEZ, M. y GARCÍA, F. J. (2005): Búsquedas en la Web Semántica: acceso eficiente a los contenidos educativos. Ponencia presentada en VI Encuentro Internacional sobre Educación, Capacitación profesional, Tecnologías de la Información e Innovación Educativa. VirtualEduca. Junio, México D.F.

MAJÓ, J. (2000): Nuevas tecnologías y educación. Consultado el 19 de Enero de 2005 en http://www.uoc.es/web/esp/articles/joan_majo.html

MARQUÉS, P. (2000): Las tics y sus aportaciones a la sociedad. Consultado el 5 Junio 2004 en http://dewey.uab.es/pmarques/tic.htm

MATTELART, A. (2002): Historia de la sociedad de la información. Barcelona, Paidós.

McLUHAN, M. (1993): La galaxia Gutenberg: génesis del "homo typographicus" Barcelona, Círculo de Lectores.

McLUHAN, M. y POWERS, B. R. (1990): La aldea global. Barcelona, Gedisa.

MONEREO, C. (coord.) (2005): Internet y competencias básicas. Barcelona, Graó.

NAVARRO, P. (2002): Información, comunicación, conocimiento y agencia en la era de la sociedad artificial. En GARCÍA, J. M. y NAVARRO, P. (Eds.). ¿Más allá de la modernidad? Las dimensiones de la información, la comunicación y sus nuevas tecnologías. Madrid, CIS. 105-133. 
OLSON, D. R. (1999): El mundo sobre el pape: el impacto de la escritura y la lectura en la estructura del conocimiento. Barcelona, Gedisa.

ONG, W. J. (1995): Orality \& Literacy: The Technologizing of the World. London, Rouledge.

PÉREZ, J. M. (2004): Alfabetización digital y educación en medios: una necesidad emergente. Consultado el 29 de Noviembre de 2004 en http://elearningeuropa.info/index.php? page $=$ doc $\&$ doc_id $=4935 \&$ doclng $=4$

PICARDO, O. (2002): Pedagogía Informacional. Enseñar y aprender en la Sociedad del Conocimiento. Consultando el 12 de Agosto del 2004 en http://www.uoc.edu/web/esp/art/uoc/opicardo0602/opicardo0602.html

TIFFIN, J. y RAJASINGHAM, L. (1997):En busca de la clase virtual: la educación en la sociedad de la información. Barcelona, Paidós.

SANCHO, J. M. (2001): Repensando el significado y metas de la educación en la sociedad de la información. El efecto fractal. En AREA, M. (Ed.) Educar en la sociedad de la información. Bilbao, Desclée de Brouwer, 37-80.

SANZ, M. (2005): Uso pedagógico de recursos y herramientas de Internet. Ponencia presentada en el III Congreso Internacional EducaRed. Noviembre, Madrid.

(C) Ediciones Universidad de Salamanca 